\title{
Interactive comment on "Assessing reliability of hydrological simulations through model intercomparison at the local scale in the Everest region" by Judith Eeckman et al.
}

\section{Anonymous Referee \#2}

Received and published: 27 September 2017

In this paper, submitted by Eeckman et al., two hydrological models are implemented on two small Nepalese basins located in the Himalayas mountain range, with the objective of comparing their simulated responses. The study is challenged by heterogeneous and sparsely instrumented basins, by the short duration of the observation time series, and by the absence of validation data in addition to streamflow. No validation of the models is performed.

General comment:

This paper suffers from the short span of the observations series, preventing any validation that would confirm the generalisation of the optimized parameters and limiting 
the analysis to two hydrological years. In the Kharikhola basin, both models have difficulties with the rainier 2014-2015 year, probably because there are many missing streamflow observations that may have helped identifying parameters more suitable to such situation. In the Tauche basin, the models somehow disagree in 2015-2016, which is dryer that the preceding one. From an operational perspective, it is normal that a model does not behave as expected all the time (and there are many reasons for it), but in the present situation the short duration prevents any generalization that may be useful to a general audience. Overall, the paper should probably be targeted towards a more regional audience, since no methodological advancements are proposed.

Major comments:

The issue of the "extreme climate heterogeneities" is central to the justification of this paper but is not documented by the authors: only basin-averaged precipitation rates are provided (Figs 4 and 5). Since the Kharikhola precipitation are captured at five locations, the authors could have explored its heterogeneity, especially since the repetitiveness of the precipitation information for that basin is questioned by the authors in page 13.

In page 3, the authors wrote that "the comparison of two models is particularly of benefit to estimate structural uncertainties in the modeling approaches". I am not convinced that comparing the annual volumes (Table 4) and the daily time series (Figs 4 and 5) of simulated variables is enough to tackle the issue of structural uncertainty. Without longer time series and verification observations in addition to streamflow, the authors are limited to identifying similitudes and differences in both model simulations.

The Base Flow Index is at best an empirical tool. I am surprised that it is used here to evaluate the quality of a land surface model (ISBA) that should reflect physical processes (page 16). I am not much more convinced that it is a good idea for the J2000 model. This needs much further justifications, including verifications in heterogeneous basins such as the Himalayas.

Printer-friendly version

Discussion paper 
It is important to know which score was used in the calibration process of both models - I guess that it is probably one of the five ones used for verification. The list of selected scores could also be improved. First, $r 2$ is not really a performance indicator and may be removed from the paper. Second, NSEsqrt is an all-purpose score without too much emphasis on low or high streamflows (Oudin et al., 2006). It does not reflect low flow performance as written in the paper. It is NSEinv that is the best option for low flow applications (Pushpalatha et al., 2012). I suggest that the authors consider it as well. Third, NSEhigh is much less common and possibly risky with short duration time series.

Minor comments:

No justifications are provided for the model selection.

The authors should clarify what they meant when writing that "a local observer indicated that the river was frozen". Was it frozen from top to bottom? Air temperature does not seem quite cold enough for it to happen. Otherwise, why impose zero flow during that time?

\section{References:}

Oudin, L., Andréassian, V., Mathevet, T., Perrin, C., Michel, C., 2006. Dynamic averaging of rainfall-runoff model simulations from complementary model parameterizations. Water Resour. Res. 42, 2005WR004636.

Pushpalatha, R., Perrin, C., Le, N., Andréassian, V., 2012. A review of efficiency criteria suitable for evaluating low-flow simulations. J. Hydrol. 420-421, 171-182.

Interactive comment on Hydrol. Earth Syst. Sci. Discuss., https://doi.org/10.5194/hess-2017401, 2017. 